\title{
Influence of Sleeve Gastrectomy on Gastro-Esophageal Reflux Disease
}

\author{
Ashraf Abd El-Hameed Abd El-Moneim, Abd El-Fattah Morsi, Tamer Abdu Mohammed \\ Megahed*
}

\author{
Department of General surgery, Faculty of Medicine, Al-Azhar University
}

*Corresponding author: Tamer Abdu Mohammed Megahed, Mobile: +201000862100, E-Mail: tamer.a.m390@gmail.com

\begin{abstract}
Background: bariatric operations can induce reflux by affecting organs motor functions depending on the type of procedure performed. Laparoscopic sleeve gastrectomy (LSG) relatively new option of morbid obesity treatment, simpler to perform, short learning curve, and shorter duration (procedure time and hospital stay).
\end{abstract}

Objectives: The aim of this work was to evaluate upper gastrointestinal symptoms before and after sleeve gastrectomy on short term follow up period of 6 months post-operatively and to assess the relation between Gastro-Esophageal Reflux Disease and sleeve gastrectomy.

Patients and Methods: This follow up study included 50 morbidly obese patients treated with laparoscopic sleeve gastrectomy during the period from May 2018 to October 2018. Rome III Criteria and upper GI Endoscopy evaluation was done preoperatively then 6 months postoperatively concerning upper GI symptoms.

Results: Before LSG 60\% asymptomatic, $40 \%$ GERD, $6.7 \%$ dyspepsia (PDS predominance). UGI endoscopy showed $40 \%$ no significant findings, $60 \%$ gastritis, $20 \%$ esophagitis, $13.3 \%$ duodenitis, DU $6.7 \% .40 \%$ H-pylori positive and $60 \%$ negative. After 6 months follow-up, $93.3 \%$ complained of upper GI symptoms, $66.7 \%$ prevalence of dyspepsia ( $p<0.001)$. GERD symptoms disappear in $83.3 \% .20 \%$ increased Vomiting of all patients associated with GERD ( $\mathrm{p}=0.030) .13 .3 \%$ increased Dysphagia $(\mathrm{p}$ $=0.125)$ associated with dyspepsia. $100 \%$ correlation between GERD and hiatus hernia $(\mathrm{p}<0.001)$ and $66.7 \%$ between vomiting and incompetent cardia $(\mathrm{p}=0.029)$.

Conclusion: Improvement of Gastro-Esophageal Reflux Disease after Sleeve Gastrectomy in patients complaining of GERD pre-operatively, although there is incidence of post-operative hiatus hernia and develop of gastrointestinal symptoms .

Recommendation: further studies have to be applied.

Keywords: Obesity, laparoscopic sleeve gastrectomy, upper gastrointestinal symptoms, GERD, dyspepsia.

\section{Introduction}

Gastro-Esophageal Reflux Disease (GERD) may present with half the obese patients need surgical intervention with high incidence between obesity and (GERD) ${ }^{(1)}$.

GERD incidence range among the obese population is $22-70 \%$. Range among non-obese population is $15-20 \%$ and Barrett's esophagus is $1-2 \%{ }^{(2)}$. So obesity is reflux pathogenesis risk factor. Bariatric operations can induce reflux alone away from body mass index (BMI) factors according to procedure performed ${ }^{(\mathbf{1})}$.

Sleeve Gastrectomy and Gastric Rouxen-Y bypass show great results in weight loss but differ in the impact on co-morbidities ${ }^{(3)}$. Gastric bypass improve the symptoms of
GERD superiorly according to databases suggestion ${ }^{(4)}$. Few studies have clams that Sleeve Gastrectomy may predispose GERD "de novo" (5).

The golden standard operation that widely used for weight loss, control of comorbidities and improve Gastro-Esophageal Reflux symptoms become Roux-en-Y Gastric bypass ${ }^{(6)}$.

Sleeve Gastrectomy originally was the first step in duodenal switch, then for its good results established a definitive procedure ${ }^{(7)}$. Sleeve Gastrectomy effect on GERD is unknown as some studies suggests that it may exacerbate or induce symptoms when done to 
previously asymptomatic patients due to the anatomical changes introduced ${ }^{(\mathbf{8})}$.

\section{Aim of Work:}

The aim of this work is to evaluate upper gastrointestinal symptoms before and after Sleeve Gastrectomy on short term follow up period of 6 months post-operatively and to assess the relation between Gastro-Esophageal Reflux Disease and Sleeve Gastrectomy.

\section{Patients and Methods}

\section{A. Patients' Selection:}

This follow up study included 50 morbid obese patients with BMI range between 38.7 to $55 \mathrm{~kg} / \mathrm{m}^{2}$ without Postprandial (>30 min) epigastric pain, Bilious vomiting, bleeding disorder, uncontrolled Diabetes Mellitus nor uncontrolled Hypertension who were treated surgically after repeating failure of weight loss after multidisciplinary medical treatment by laparoscopic sleeve gastrectomy during the period from May 2018 to October 2018. Rome III Diagnostic Criteria and upper GastroIntestinal Endoscopy evaluation were done preoperatively and 6 months postoperatively concerning upper gastrointestinal symptoms.

\section{B. Methods:}

\section{I- Preoperative evaluation by:}

History taking, general examination and complete laboratory investigation.

\section{II- Preoperative examination:}

1. Rome III Diagnostic Criteria evaluation by filling out the validated

Rome III symptomatic questionnaire.

2. Waist circumference.

3. Waist to hip ratio.

III- Preoperative surgical investigation:

1. H-pylori antigen rapid test (HPSA).

2. Chest X-ray.

3. Pulmonary function tests.

4. Upper GI endoscopy.

\section{IV- Post operative follow up:}

Gastrograffin upper gastro-intestinal contrast study on postoperative day 1-5 to evaluate the gastric volume and to exclude postoperative leak was done.

Rome III Diagnostic Criteria (Ref) evaluation by filling out the validated Rome III symptomatic questionnaire and upper Gastro-Intestinal Endoscopy was done 6 months postoperatively concerning upper gastrointestinal symptoms using for the study to detect post-operative complications related to upper gastrointestinal symptoms for the study.

\section{Statistical analysis:}

Recorded data were analyzed using the statistical package for social sciences, version 20.0 (SPSS Inc., Chicago, Illinois, USA). Quantitative data were expressed as mean \pm standard deviation (SD). Qualitative data were expressed as frequency and percentage. 


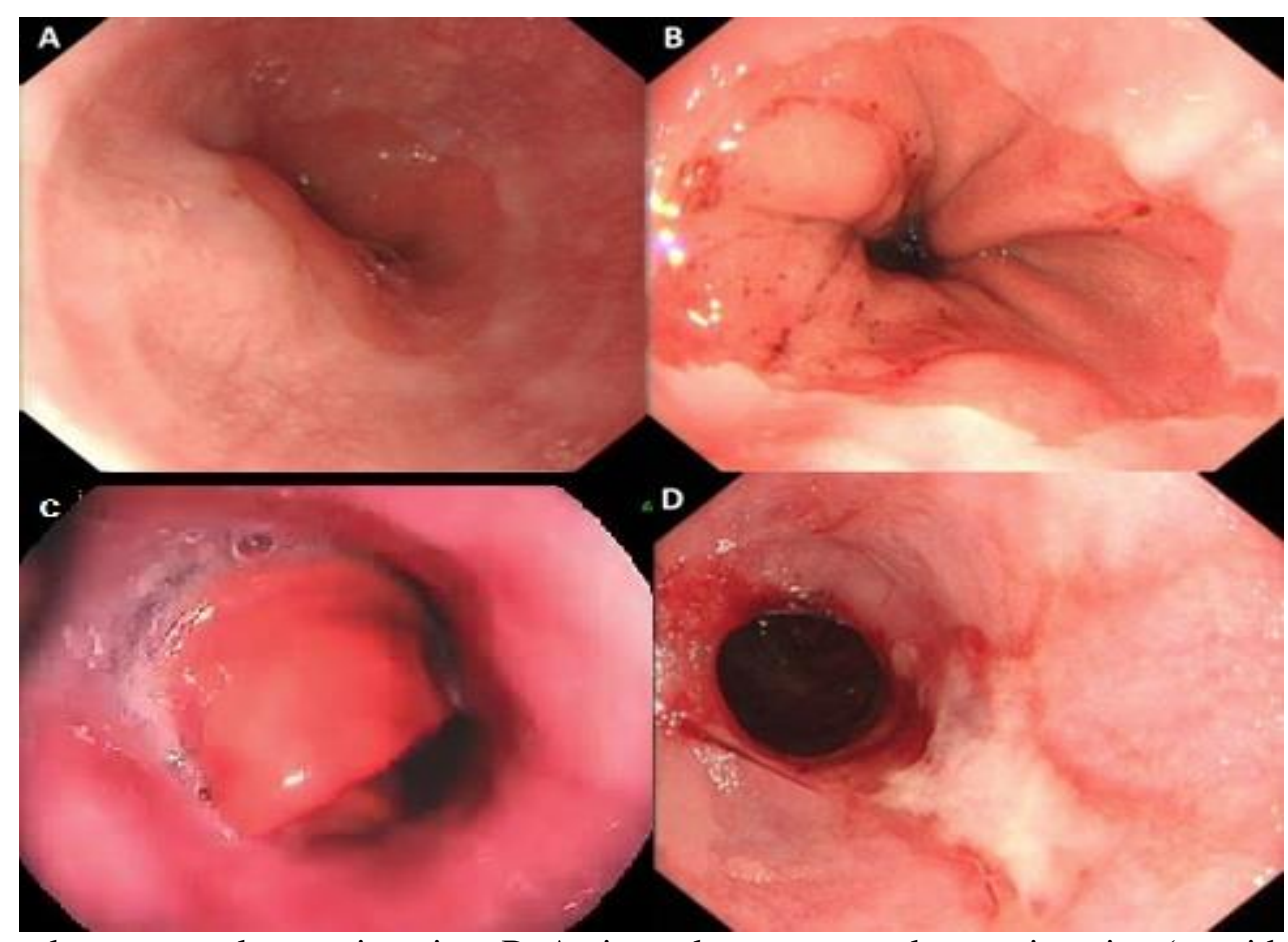

A. Normal squamo-columnar junction; B. An irregular squamo-columnar junction (considered within normal limits); C. Sliding Hiatus Hernia; D. Reflux esophagitis with impaired visualisation of the lower esophagus

Figure (1): Endoscopic appearances of the effect of Reflux on esophagus

\section{Results}

Table (1): Distribution of studied patients regarding their risk factors

\begin{tabular}{|c|c|c|c|}
\hline \multicolumn{2}{|c|}{ Risk factors } & No of patients & Percentage \\
\hline \multirow{2}{*}{ DM } & positive & 3 & $6 \%$ \\
\cline { 2 - 4 } & negative & 47 & $94 \%$ \\
\hline \multirow{2}{*}{ HTN } & Positive & 7 & $14 \%$ \\
\cline { 2 - 4 } & Negative & 43 & $86 \%$ \\
\hline \multirow{2}{*}{ Asthma } & Positive & 4 & $8 \%$ \\
\cline { 2 - 4 } & negative & 46 & $92 \%$ \\
\hline
\end{tabular}

Table (2): Distribution of studied patients regarding their gender

\begin{tabular}{|c|c|c|c|}
\hline Gender & Female & Male & Total \\
\hline No of patients & 36 & 14 & 50 \\
\hline
\end{tabular}

Table (3): Distribution of studied patients regarding their gender and BMI

\begin{tabular}{|c|c|c|c|}
\hline BMI & Female & Male & Total \\
\hline Range $(\mathbf{K g} / \mathbf{m} 2)$ & $33-55$ & $41-54$ & $33-55$ \\
\hline Median $(\mathbf{K g} / \mathbf{m} 2)$ & 49.15 & 47 & 48.7 \\
\hline
\end{tabular}

Table (4): Distribution of studied patients regarding their percent of excess weight loss (\%EWL)

\begin{tabular}{|c|c|c|c|}
\hline EWL\% & Female & Male & Total \\
\hline Mean & $66.5 \%$ & $68.8 \%$ & $67 \%$ \\
\hline
\end{tabular}

Table (5): Relation between upper GI symptoms pre and post opertaive

\begin{tabular}{|c|c|c|c|c|c|}
\hline \multirow[t]{2}{*}{ Symptoms } & \multicolumn{2}{|c|}{ Pre } & \multicolumn{2}{|c|}{ Post } & \multirow[t]{2}{*}{ P value } \\
\hline & NO. & $\%$ & NO. & $\%$ & \\
\hline Free & 30 & $60.00 \%$ & 3 & $6.00 \%$ & $<0.001 \mathrm{~S}$ \\
\hline GERD & 20 & $40.00 \%$ & 13 & $26.00 \%$ & 0.454 \\
\hline Dyspepsia & 3 & $6.00 \%$ & 33 & $66.00 \%$ & $<0.001 \mathrm{~S}$ \\
\hline Vomiting & 0 & $0.00 \%$ & 10 & $20.00 \%$ & 0.030 \\
\hline Dysphagia & 0 & $0.00 \%$ & 7 & $14.00 \%$ & 0.125 \\
\hline
\end{tabular}


Sixty percent (30/50) of patients had no symptoms, $40 \%$ had symptoms of GERD $20 / 50$ in the form of heart burn, regurgitation or cough, 3/20 had dyspepsia in the form of post prandial distress (PPD) or epigastric pain syndrome (EPS) accompanied with GERD $6 \%$.

Among our study group of patients who were all subjected to upper GI endoscopy preoperative 20/50 of patients had no specific finding, $40 \%$ where $30 / 50$ had gastritis $60 \%$. $20 \%$ had associated oesophagitis 10/30, 14\% had duodenitis $7 / 30$ and $6 \%$ were accompanied by duodenal ulcers $3 / 30$.

After 6 months of follow up of upper GI symptoms there was a significant increase in the number of patients developing upper GI symptoms $94 \%$ (47/50) compared to $40 \%$ $(20 / 50)$ in the preoperative period $\mathrm{p}<0.001$. Only $6 \%$ of patients had No symptoms in the postoperative period $3 / 50$ compared with $60 \%$ of patients had no symptoms preoperatively.

The percentage of patients who developed GERD did not significantly increase $\mathrm{p}=0.454$ with post-operative percentage of $26 \% 13 / 50$ compared to $40 \% 20 / 50$ in the pre-operative period. Patients complaining of dyspepsia increased significantly in number $\mathrm{p}<0.001$ with $66 \% 33 / 50$ compared to $6 \% 3 / 50$ preoperatively. New symptoms developed and persist throughout the follow up period like vomiting which increased significantly reaching $20 \% \mathrm{p}=0.030$ of all patients all associated with GERD 10/13 and dysphagia non-significantly $\mathrm{p}=0.125$ with a percentage of $14 \%$ associated with dyspepsia. 7/13

The prevalence of GERD was not significantly with post-operative percentage of $26 \%(13 / 50)$ compared to $40 \%(20 / 50)$ in the pre-operative period $(\mathrm{p}=0.454)$. Whereas only $30.77 \%$ (4/13) of patients who developed GERD post operatively already had GERD symptoms in the pre-operative period $20 \%$ (4/20) of patients with GERD before LSG. The symptoms of GERD newly developed in $18 \%$ (9/50) and pre-operative GERD symptoms disappeared in $80 \%(16 / 20)$. 
The prevalence of dyspepsia increased significantly in the post-operative period with $66 \%(33 / 50)$ compared to $6 \%(3 / 50)$ preoperatively $(\mathrm{p}<0.001)$ whereas $10 \%(3 / 33)$ of patients who developed dyspepsia post operatively already had dyspepsia in the preoperative period $(100 \%(3 / 3)$ of patients with dyspepsia before LSG) and 90\% (30/33) developed De-novo dyspepsia.

Vomiting was a persistent newly developed symptom in the post-operative period with a significant value 20\% (10/50) (p $=0.030)$ where all were associated with GERD (6/8).

Another newly developed symptom after LSG was dysphagia with a non-significant value $14 \%(7 / 50)(\mathrm{p}=0.125)$.

On performing UGI endoscopy 6 months post-operatively on all study subjects $20 \%$ (10/50) came negative for findings, $46 \%$ (23/50) showed gastritis, 26\% (13/50), showed incompetent cardia and 34\% (17/50) showed Hiatus hernia. Among the study group of patients a significant relation was found between positive symptoms after 6 month of follow up post-operatively and upper GI endoscopy done after 6 months for correlation with symptoms where $85.1 \%$ (40/47) of the patients having troublesome symptoms had positive findings and $100 \%$ (3/3) of patients who had no symptoms had no obvious finding ( $\mathrm{p}=0.034)$.

A significant relation was found between patients complaining of GERD symptoms 6 months post-operatively and the finding of Hiatus Hernia on upper GI endoscopy where $100 \%(17 / 17)$ rendered positive for this finding $(\mathrm{p}<0.001)$.

A significant relation was found between patients complaining of vomiting 6 months post-operatively and the finding of incompetent cardia on upper GI endoscopy where $70 \%(7 / 10)$ rendered positive for this finding $(\mathrm{p}=0.029)$.

The mean for patients with no comorbidities in relation to BMI extent was 47.01 (SD 5.31) where for patients with HTN was 52.7, patients with diabetes was 51.55 (SD 3.29) and patients with asthma was 40.6 with no significant correlation between them ( $\mathrm{p}$ $=0.461$ ) 
Table (6): Relation between BMI and co-morbidities

\begin{tabular}{|c|c|c|c|}
\hline Comorbidity & \multicolumn{2}{|c|}{ BMI } & \multirow{2}{*}{ P VALUE } \\
\hline & Mean & Standard Deviation & \\
\hline Negative & 47.01 & 5.31 & \multirow{2}{*}{0.461} \\
\hline HTN & 52.70 & 0.00 & \\
\hline DM & 51.55 & 3.29 & \\
\hline Asthma & 40.60 & 0.00 & \\
\hline
\end{tabular}

A significant correlation was found between H-pylori infection and GERD preoperatively $65 \%(13 / 20)(\mathrm{p}=0.024)$ whereas No significant correlation was found between patients with dyspepsia and $\mathrm{H}$ pylori infection proven by stool antigen testing ( $\mathrm{p}=0.152)$. Patients marked negative for symptoms were also marked negative for H-pylori infection with percentage of 76.6\% $(23 / 30)$ with significant value $(\mathrm{p}=0.024)$.

No significant correlation was found between special habits of medical importance which is exclusively smoking in our study and upper GI symptoms before LSG.

\section{Discussion}

Obesity continues to be a leading public health concern associated with many comorbidities that significantly decrease life expectancy ${ }^{(\mathbf{1 0})}$.

Middle East countries in general are typical developing countries that have experienced a rapid rise in the prevalence of morbid obesity. According to the World Health Organization, obesity has reached an 'alarming level'. The same study pointed out that unlike Europe and North America, obesity is more prevalent among women and in urban areas in the Middle East ${ }^{(11)}$.

Weight reduction can be achieved by several non-surgical methods that include; diet control, physical exercise, and/or drug therapy but these methods often elicit compensatory changes in appetite and energy expenditure that make weight loss of more than 5 to 10 percent unlikely to be sustained for more than 5 years ${ }^{(12)}$.

In contrast, surgery typically causes substantial long term sustained weight loss (13).
In the same aspect, the rising prevalence of morbid obesity and super-obese patients $\left(\mathrm{BMI}>50 \mathrm{Kg} / \mathrm{m}^{2}\right)$ who are seeking treatment had led to that surgery became the choice which provides adequate EWL in comparison to non-surgical methods ${ }^{(14)}$.

Over the last 20 years, bariatric surgery has come to play a significant role in confronting this problem, using either restrictive or mixed restrictive and malabsorptive techniques ${ }^{(\mathbf{1 5})}$.

Lapasroscopic Sleeve Gastrectomy remains one of the safest and most effective modern surgical options for the treatment of morbid obesity ${ }^{(10)}$.

Gastroesophageal reflux disease (GERD) is one of the most prevalent chronic gastrointestinal diseases, with an estimated $20-30 \%$ of the US adult population experiencing heartburn or acid regurgitation or both at least once a week Obesity is considered a major risk factor in the pathogenesis of GERD, and approximately $50 \%$ of morbidly obese patients have signs or symptoms of GERD ${ }^{(\boldsymbol{9})}$.

Also epigastric pain syndrome and post prandial distress dyspepsia are present in 28 and $11 \%$ of the obese patients considered for bariatric surgery ${ }^{(16)}$.

Data from large databases suggest that all of the common bariatric procedures usually improve GERD symptoms, with Roux-en-Y gastric bypass being superior to Laparoscopic adjustable gastric banding and Laparoscopic sleeve gastrectomy in this regard. Yet, smaller prospective studies indicate that LSG can induce de-novo GERD in some patients ${ }^{(9)}$. 
The removal of the gastric fundus, a large part of the body and a portion of the antrum, leads to important anatomical and functional alterations that affect both gastric acid secretion and motility, in particular accommodation, which may give rise to gastrointestinal GI symptoms ${ }^{(17)}$.

In our study 50 patients ( 36 females and 14 males) with mean age of $38.4 \pm 7.4(36.2$ for females and 40.3 for males) and BMI of $42.9 \mathrm{~kg} / \mathrm{m}^{2} \pm 7.6$ (43 for females and 41 for males) with 3 patients with DM (6\%), 7 with HTN (14\%) and 4 with asthma (8\%). Twenty patients tested +ve for H-pylori (40\%) and took medications for eradication for 2 weeks.

In the preoperative assessment with Rome III criteria questionnaire and UGI endoscopy $60 \%$ had no UGI symptoms, 40\% had GERD and 6\% had dyspepsia. All were subjected to UGI endoscopy and showed no significant finding in $40 \%$, gastritis in $60 \%$, esophagitis in $20 \%$, duodenitis in $14 \%$ and DU in 6\%. A significant relation was found between GERD as a symptom and H-pylori infection $(\mathrm{p}=0.024)$ and another strong relation was found between gastritis in preoperative UGI endoscopy and H-pylori infection 66.7\% $(20 / 30)(p<0.001)$ and between duodenitis and H-pylori infection $100 \%$ (7/7) $(\mathrm{p}=0.018)$.

In a comparative study performed UGI endoscopy on 142 morbidly obese patients who filled out the validated Rome III symptomatic questionnaire preoperatively. Symptoms were referred by $43 \%$ of patients: gastroesophageal reflux disease (GERD) $27.9 \%$ and dyspepsia $24.6 \%$, subdivided in postprandial distress (PDS) $66.7 \%$ and epigastric pain $33.3 \%$ syndromes. Of GERD patients, $19.7 \%$ presented concomitantly PDS. Belching was present in $8.2 \%$ and nausea and/or vomiting in $1.6 \%$ of patients. At endoscopy, one or more lesions were present in $47.1 \%$ of the patients: erosive esophagitis $5.6 \%$, hiatal hernia $23.2 \%$, gastroduodenal erosions $6.3 \%$, and peptic ulcers $3.5 \%$. At histology, $24 \%$ of patients have Helicobacter pylori infection, and its prevalence in gastroduodenal erosions and ulcers was 22.2 and $60 \%$, respectively. $\mathrm{He}$ stated that the presence of symptoms cannot be considered as a valuable guide to indicate endoscopy since the majority of endoscopic lesions were asymptomatic and not $\mathrm{H}$. pylori related ${ }^{(\mathbf{1 8})}$.

EWL\% after 6 months from LSG showed a mean of $67 \% \pm 4.58 \%(66.5 \%$ for females and $68.8 \%$ for males). in a comparative study who compared between LRYGP and LSG where 34 patients had LSG (28f and $6 \mathrm{~m}$ ) and achieved EWL\% mean of $46.6 \% \pm 16.1 \%$ after 6 months of follow up ${ }^{(19,20)}$.

In comparative studies maximum weight loss was in the first 6 months, leveling off at 1 year in the majority of cases with satisfactory and long term effects regarding weight loss in our study and comparative ones.

After a median follow up of 6 months duration there have been significant development of UGI symptoms mainly dyspepsia in the form of PPD and only 3 patients with EPS. De-novo dyspepsia was observed in 66\% mostly in the form of PPD, where the prevalence of dyspepsia was highly significant $(\mathrm{p}<0.001)$. The prevalence of GERD was not statistically different before and after LSG but GERD symptoms was observed to disappear gradually in $880 \%$ of patients who had considerable GERD symptoms pre-operatively and newly developed in $34 \%$. Hiatus hernia was a significant finding post LSG in association with GERD were $100 \%$ of patients complaining of GERD had hiatus hernia at endoscopy $(\mathrm{p}<0.001)$ representing $34 \%$ of all findings reported by endoscopy after LSG.

In a comparable study which applied the same questionnaire in our study (Rome III criteria) for UGI symptoms on 74 patients with a median follow up of 13 months. LSG was associated with de novo dyspepsia-like syndrome, with an (OR of $7.0095 \%$ CI 2.9$18.3, \mathrm{p}<0.0001)$. In turn, the prevalence of GERD before and after surgery was not different $(\mathrm{OR}=1.083,95 \%$ CI 0.4652 2.530, $\mathrm{p}>0.05)$. Pre-surgical GERD symptoms disappeared in $65 \%$ after LSG, worsen or did not change in $35 \%$, and newly developed in $22 \%^{(16)}$. 
In another study on 28 patients mean BMI of $55.5 \mathrm{~kg} / \mathrm{m}^{2}$ using the GERD score questionnaire, all patients were interviewed to evaluate their reflux symptoms had a $64 \%$ response rate, with $22 \%$ of patients indicating new-onset GERD symptoms despite receiving daily anti reflux therapy (21).

More than one of the pathophysiological mechanisms underlying functional dyspepsia may be involved after LSG ${ }^{(22)}$.

Limited gastric accommodation related to fundus removal may be one of the major factors since the new stomach has a greater luminal pressure and smaller volume, having only one tenth of the distention. Altered duodenal sensitivity to nutrients could be worsened by faster gastric emptying, and this might promote dyspeptic symptoms ${ }^{(23)}$.

It can then be speculated that the mechanism of upper GI symptoms after LSG is mainly due to altered motility patterns rather than acid-related disorders. Some evidence strengthens this hypothesis: the association between dysphagia and LSG, its lack of association with GERD, and the reduced efficacy of PPI on post-surgical GI symptoms ${ }^{(16)}$.

Vomiting was also a significant symptom in our study that appeared totally de-novo after LSG in $20 \%$ of the study figures and continues to be a troublesome symptom for the whole follow up period almost always associated with GERD, it was mildly responsive to repetition of PPI therapy and was associated with incompetent cardia at endoscopy in $66.7 \%$ $(\mathrm{p}=0.029)$. Dysphagia also developed denovo in $14 \%$ of patients post LSG and was entirely associated with dyspeptic symptoms.

A comparative study found out that dysphagia newly developed after LSG in $19.7 \%$ of the patients to both solid and fluid in $85.7 \%$ of them and was mainly associated with PDS-like dyspepsia rather than GERD, while vomiting was associated with $13.6 \%$ of dyspeptic patients. Dysphagia in this surgical setting has been ascribed to impaired emptying of the gastric pouch rather than to alteration of esophageal motility. It is, however, to be noted that this symptom might also be due to the patient's interpretation of feeling full due to the lower distend ability of the neo-stomach. Besides, upper discomfort after LSG might also be influenced by technical pitfall as the distance from pylorus (i.e., portion of antrum resected) and the bougie used to calibrate the sleeve ${ }^{(\mathbf{1 6})}$.

Concerning postoperative UGI endoscopy findings and relation to symptoms that developed post LSG, our study found that $20 \%$ were -ve for findings, $46 \%$ had gastritis, $26 \%$ had incompetent cardia and $34 \%$ had hiatus hernia. $85.1 \%$ of patients with symptoms had +ve findings $(\mathrm{p}=0.034)$ where a significant correlation was found between GERD and hiatus hernia $100 \% \quad(\mathrm{p}<0.001)$ and vomiting and incompetent cardia $66 \% \quad(\mathrm{p}=0.029)$. The finding of esophagitis, duodenitis and DU disappeared post-operatively.

In a comparative study UGI endoscopy was done preoperatively and postoperatively at six months. Preoperatively six $18.8 \%$ patients had esophagitis with $50 \%$ being LA grade B. Postoperatively the incidence of esophagitis increased to $25 \%$, but this rise was not statistically significant. Moreover, there was improvement in five patients in terms of reduction of LA grading including one patient with resolution of esophagitis. $34.4 \%$ of patients shows hiatal hernia, all small, in postoperative period (24). Another study revealed that the occurrence of erosive esophagitis after LSG is related to the presence of hiatal hernia after the operation. GERD symptoms are not consistent with the presence of erosive esophagitis as $40.1 \%$ of patients who did not have postoperative GERD symptoms still had postoperative erosive esophagitis on endoscopy. Therefore, he proposes that postoperative follow-up endoscopy is necessary to identify the true prevalence of postoperative GERD ${ }^{(25)}$.

\section{Conclusion}


This study suggests that LSG represents a safe and effective procedure to achieve significant weight loss with satisfactory results to patients undergoing the procedure. LSG showed no significant change in GERD prevalence which was considered to be a main troublesome, as only one third of patients who developed GERD post operatively already had GERD symptoms in the pre-operative period, on the other hand GERD symptoms newly developed in few number of the patients and pre-operative GERD symptoms improved in most of patients with real and significant impact on upper gastrointestinal symptoms mainly dyspepsia which increased in prevalence and incidence postoperatively, on the other hand significant association between GERD and vomiting was found also with a significant correlation to incompetent cardia in UGI endoscopy. A consistent relation was found between GERD and the finding of hiatus hernia in the postoperative setting raising the question of additive or alternative procedures that could prevent the incidence of hiatus hernia postoperatively.

\section{Recommendation}

Further studies have to be applied to a larger number of patients for a longer period of follow up.

\section{References}

1. Prachand VN, Alverdy JC (2010): Gastroesophageal reflux disease and severe obesity: Fundoplication or bariatric surgery? World J. Gastroenterol., 16(30):3757-61.

2. Friedenberg FK, Xanthopoulos M, Foster GD et al. (2008): The association between gastroesophageal reflux disease and obesity. Am. J. Gastroenterol., 103(8):2111-22.

3. Yaghoubian A, Tolan A, Stabile BE et al. (2012): Laparoscopic Roux-en-Y gastric bypass and sleeve gastrectomy achieve comparable weight loss at 1 year. Am. Surg., 78(12):1325-8.

4. Pallati PK, Shaligram A, Shostrom VK et al. (2014): Improvement in gastroesophageal reflux disease symptoms after various bariatric procedures: review of the bariatric outcomes longitudinal database. Surg.
Obes. Relat. Dis., 10(3):502-7.

5. Del Genio G, Tolone S, Limongelli $P$ et al. (2014): Sleeve gastrectomy and development of "de novo" gastroesophageal reflux. Obes. Surg., 24(1):71-7.

6. Buchwald H, Buchwald JN (2002): Evolution of operative procedures for the management of morbid obesity 19502000. Obes. Surg., 12(5):705-17.

7. Gagner M, Deitel M, Erickson AL et al. (2013): Survey on laparoscopic sleeve gastrectomy (LSG) at the Fourth International Consensus Summit on Sleeve Gastrectomy. Obes. Surg., 23(12):2013-7.

8. Elder KA, Wolfe BM (2007): Bariatric surgery: a review of procedures and outcomes. Gastroenterology, 132(6):2253-71

9. Tutuian R (2014): Effects of bariatric surgery on gastroesophageal reflux. Curr. Opin. Gastroenterol., 30(4):434-8.

10. Jackson TD, Hutter MM (2012): Morbidity and effectiveness of laparoscopic sleeve gastrectomy, adjustable gastric band, and gastric bypass for morbid obesity. Adv. Surg., 46:255-68.

11. Musaiger AO, Al-Awadi AH and AlMannai MA (2000): Lifestyle and social factors associated with obesity among the Bahraini adult population. Ecol. Food and Nut.r, 39: 121-133.

12. Bray G, Tartaglia L (2000): Medicinal strategies in the treatment of obesity, Nature, 404: 672-7.

13. Mun E, Blackburn G, Matthews J (2001): Current status of medical and surgical therapy for obesity, Gastroenterology, 120: 66981.

14. Bassiony F, Fouad A, Abolfotooh A et al. (2009): Laparoscopic sleeve gastrectomy as a sole procedure for morbid obesity. Kasr El Aini J. Surg., 10 (1): 23-30.

15. Buchwald $\mathbf{H}$, Avidor $\mathbf{Y}$, Braunwald $\mathbf{E}$ et al. (2004): Bariatric surgery: a systematic review and meta-analysis. 
JAMA., 292(14):1724-1737.

16. Carabotti M, Silecchia G, Greco F et al. (2013): Impact of laparoscopic sleeve gastrectomy on upper gastrointestinal symptoms. Obes. Surg., 23(10):1551-7.

17. Santoro $S$ (2007): Technical aspects in sleeve gastrectomy. Obes. Surg., $17: 1534-5$.

18. Carabotti M, Avallone M, Cereatti F et al. (2016): Usefulness of Upper Gastrointestinal Symptoms as a Driver to Prescribe Gastroscopy in Obese Patients Candidate to Bariatric Surgery. A Prospective Study. Obes. Surg., 26(5):1075-80.

19. Albeladi B, Bourbao-Tournois $\mathrm{C}$ and Huten N (2013): Short- and midterm results between laparoscopic roux-en-y gastric bypass and laparoscopic sleeve gastrectomy for the treatment of morbid obesity. J. Obes., 2013: 634-653.

20. Dapri G, Vaz C, Cadière GB et al. (2007): A prospective randomized study comparing two different techniques for laparoscopic sleeve gastrectomy. Obes. Surg., 17 (11): 1435-41.
21. Howard DD, Caban AM, Cendan JC et al. (2011): Gastroesophageal reflux after sleeve gastrectomy in morbidly obese patients. Surg. Obes. Relat. Dis., 7:709-13.

22. Oustamanolakis P, Tack J (2012): Dyspepsia: organic versus functional. J. Clin. Gastroenterol., 46:175-90.

23. Yehoshua RT, Eidelman LA, Stein M et al. (2008): Laparoscopic sleeve gastrectomy - volume and pressure assessment. Obes. Surg., 18:1083-8.

24. Sharma A, Aggarwal S, Ahuja V et al. (2014): Evaluation of gastroesophageal reflux before and after sleeve gastrectomy using symptom scoring, scintigraphy and endoscopy. Surg. Obes. Relat. Dis., 14:1-6.

25. Tai CM, Huang CK (2013): Increase in gastroesophageal reflux disease symptoms and erosive esophagitis 1 year after laparoscopic sleeve gastrectomy among obese adults. Surg. Endosc., 27:1260-6. 